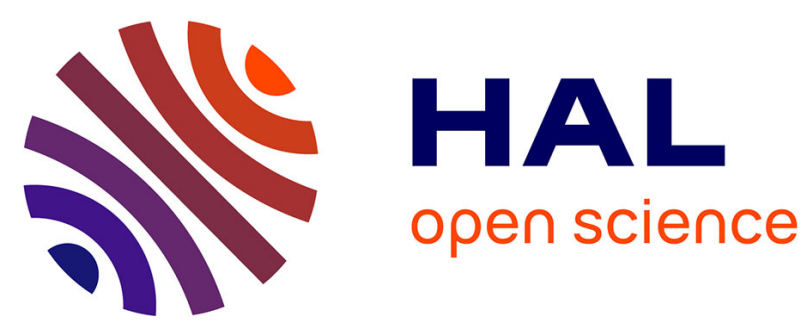

\title{
Spree-Athen nach dem Untergang. Eduard Meyer zur Parallelität von Geschichte
}

\author{
Christian Wendt
}

\section{To cite this version:}

Christian Wendt. Spree-Athen nach dem Untergang. Eduard Meyer zur Parallelität von Geschichte. KTÈMA Civilisations de l'Orient, de la Grèce et de Rome antiques, 2017, 42, pp.151-166. halshs01670126

\section{HAL Id: halshs-01670126 \\ https://shs.hal.science/halshs-01670126}

Submitted on 21 Dec 2017

HAL is a multi-disciplinary open access archive for the deposit and dissemination of scientific research documents, whether they are published or not. The documents may come from teaching and research institutions in France or abroad, or from public or private research centers.
L'archive ouverte pluridisciplinaire HAL, est destinée au dépôt et à la diffusion de documents scientifiques de niveau recherche, publiés ou non, émanant des établissements d'enseignement et de recherche français ou étrangers, des laboratoires publics ou privés. 

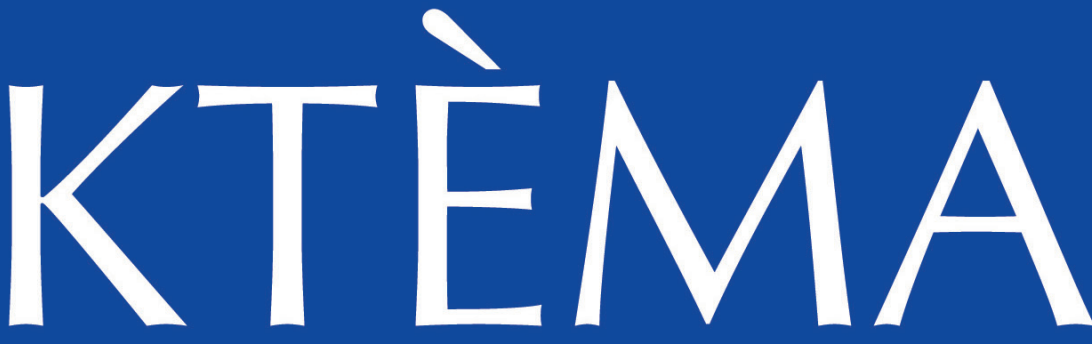

\section{CIVILISATIONS DE L'ORIENT, DE LA GRÈCE ET DE ROME ANTIQUES}

Edith Foster

Edmond LÉvY

Tim Roop

Cinzia Bearzot

\section{Michel Нumm}

David S. Levene

Estelle OudoT

Hans Kopp

Maciej JUNKIERT

Tobias Јоно

Christian WENDT

Oliver SCHELSKe

Dominique Lenfant

Neville MORLEY

Agnès Molinier Arbo

Michèle Coltelloni-Trannoy Philippe Torrens

Jean Ducat

Michel WoronofF

\section{Les interprétations de la défaite de 404}

Interpretations of Athen's defeat in the Peloponnesian war............................................................ 7

Thucydide, le premier interprète d'une défaite anormale .............................................................. 9

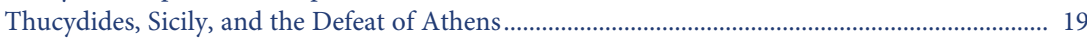

La $\sigma u \mu \varphi$ ód de la cité

La défaite d'Athènes (405-404 av. J.-C.) chez les orateurs attiques.............................................. 41

Rome, une «cité grecque» prise par les Hyperboréens.................................................................... 53

Rome Redeems Athens?

Livy, the Peloponnesian War, and the Conquest of Greece............................................................ 73

Ultime défaite d'Athènes ou sa plus belle victoire?

Stratégies rhétoriques autour de la bataille d'Aigos-Potamoi

dans le Panathénaïque d'Aelius Aristide

The Defeat of Athens in 404 BC in The Federalist ............................................................................ 97

Polish Reflections: The Reception of the Defeat of Athens in

the Works of Gottfried Ernst Groddeck and Joachim Lelewel.........................................................115

The Internal Commotion of Greek Culture: Jacob Burckhardt

on the Defeat of Athens in the Peloponnesian War.

Spree-Athen nach dem Untergang

Eduard Meyer zur Parallelität von Geschichte

Der Kampf um die Demokratie

Thukydides in Deutschland nach dem Ersten Weltkrieg

Défaite militaire et révolution antidémocratique

Le parallèle entre l'Athènes de 404 et la France de 1940

dans Les Oligarques de Jules Isaac.

Thucydides and the Historiography of Trauma.

\section{Le thauma dans l'historiographie grecque d'époque impériale}

Hérodien, Rome et le spectacle du pouvoir

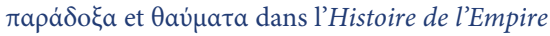

après la mort de Marc Aurèle

La place du thauma dans l'Histoire romaine de Cassius Dion............................................................219

Le lexique de l'étonnement chez Appien. Quelques remarques .......................................................23

\section{Varia}

Du caractère «mixte» du régime spartiate 251

L’image de la défaite dans l'Iliade. 


\author{
KTÈMA \\ CIVILISATIONS DE L'ORIENT, DE LA GRÈCE ET DE ROME ANTIQUES \\ Revue annuelle \\ Fondateurs : Edmond Frézouls † \\ Edmond LÉvY
}

Comité Directeur: Dominique Beyer, Bruno Bleckmann, Jean-François Bommelaer, Frédéric Colin, MireilleCorbier, GérardFreyburger, Jean Gascou, Jean-Georges Heintz, Michel Humbert, Anne Jacquemin, Stavros Lazaris, Dominique Lenfant, Edmond Lévy, Jean-Claude Margueron, Henriette Pavis d'Escurac, Laurent Pernot, Thierry Petit, Gérard SiEBERT

Rédaction:Edmond LÉvy

Dominique Beyer et Gérard Freyburger

Maquette et mise en page: Ersie LERIA

\title{
Éditeur
}

Presses universitaires de Strasbourg

5 allée du Général Rouvillois - CS50008

FR-67083 Strasbourg Cedex

Tél: (33) 0368856265

info.pus@unistra.fr

pus.unistra.fr

\section{Ventes au numéro}

En librairie ou en commande en ligne sur le site du Comptoir des presses d'universités: www.lcdpu.fr

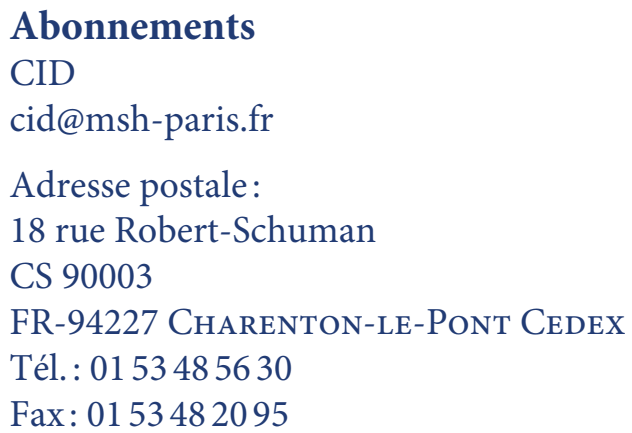




\section{Spree-Athen nach dem Untergang Eduard Meyer zur Parallelität von Geschichte}

RÉsumé-. Eduard Meyer, le grand historien de l'Antiquité et professeur à l'université Friedrich-Wilhelm de Berlin, figure parmi les intellectuels allemands de droite qui furent très actifs dans le débat politique aux alentours de la première guerre mondiale. Lors de sa prise de fonctions comme recteur de son université après la guerre, il tient un discours remarquable, qui construit un parallèle plus qu'étroit entre l'Athènes défaite de 404 et l'Allemagne battue de 1918. Dans son développement, Meyer esquisse l'idée d'un patrimoine culturel commun à Berlin et Athènes, qui légitimerait que l'Allemagne occupe le premier rang parmi les nations même dans la défaite. Cette idée repose sur une image des universités et de la vie intellectuelle allemandes comme étant les dernières valeurs indestructibles qui permettent à Meyer d'espérer un redressement de l'Allemagne tout à fait semblable à celui d'Athènes au IV siècle av. J.-C. Pour construire son discours, Meyer se sert de son grand modèle, Thucydide. Il exploite tout particulièrement l'oraison funèbre de Périclès pour souligner les valeurs qui constituent l'essence d'une société. La volonté de suivre le maître infaillible de l'historiographie et la cohérence avec laquelle Meyer utilise l'auteur athénien présentent un exemple fascinant de transformation de l'Antiquité et de réception de la défaite d'Athènes. C'est d'autant plus intéressant que Meyer n'est pas une exception en son temps, Thucydide servant de référence à d'autres intellectuels européens qui essaient d'analyser leur expérience.

Zusammenfassung-. Eduard Meyer, der berühmte Historiker der Alten Welt und Professor an der Friedrich-Wilhelms-Universität zu Berlin, zählt zu den deutschen Intellektuellen der politischen Rechten, die im Umfeld des Ersten Weltkriegs eine rege politische Publizistik betrieben. Nach dem Krieg zum Rektor seiner Universität ernannt, hält er eine bemerkenswerte Rede, in der er eine mehr als enge Parallele zwischen dem geschlagenen Athen von 404 und dem Deutschen Reich von 1918 herstellt. Meyer entwirft in seiner Gedankenführung die Idee eines gemeinsamen kulturellen Erbes für Berlin und Athen, die selbst in der Niederlage noch eine Führungsrolle für Deutschland legitimieren kann. Sie beruht auf dem Bild der deutschen Universitäten und dem deutschen Geistesleben als den letzten unzerstörbaren Werten, die Meyer auf einen Wiederaufschwung Deutschlands hoffen lassen, ganz ähnlich dem Athens im 4. Jahrhundert v.Chr. Meyer bedient sich seines großen Vorbilds Thukydides, um seine Rede zu konstruieren. Dabei greift er besonders auf die berühmte perikleische Leichenrede zurück, um die Werte zu unterstreichen, die den Kern einer Gesellschaft ausmachen. Sowohl der Wunsch, dem unfehlbaren Meister der Geschichtsschreibung nachzufolgen, als auch die Konsequenz, mit der Meyer den athenischen Autor als Vorlage verwendet, hinterlassen ein faszinierendes Beispiel für Antikentransformation und die Rezeption der Niederlage Athens. Besondere Relevanz gewinnt dies vor dem Hintergrund, daß Meyer keine Ausnahme in seiner Zeit ist, sondern eine Zahl öffentlicher Intellektueller sich des Thukydides als besondere Referenz bedienen, wenn sie versuchen, das Erlebte zu analysieren. 
Die Niederlage Athens im sogenannten Peloponnesischen Krieg, all den großen athenischen Ambitionen ein schmerzliches, wenn auch nur vorübergehendes Ende bereitend, war seit jeher eines der faszinierendsten vermeintlichen Paradoxa der antiken Geschichte. Die aufstrebende Polis mit ihrer großen Machtfülle, ihren kulturellen Errungenschaften und ihrer neuartigen bürgerschaftlichen Ordnung schließlich auf Gedeih und Verderb ihren Widersachern ausgeliefert zu sehen, der gewaltigen Flotte beraubt, die Sicherheit und Abschottung garantierenden Mauern geschleift - das Konvolut aus Innovationen, Leistungen und Erfolgen schuf eine Fallhöhe für die Polis, die gerade in der Rezeption zu einem Stoff geriet, um Hybris und hochfliegende Träume kritisch zu reflektieren und Athen zu einem wie gegebenen Exempel für die Problematik der Überdehnung eigener Machtansprüche werden zu lassen.

Die Verarbeitung dieser Katastrophe durch die Zeitgenossen, durch die Rückschau der attischen Redner und vor allem durch die Analyse ihrer Genese bei Thukydides, besaß bereits früh das Potential des Paradigmas, des warnenden Menetekels, welche politischen Fehler grundlegend für den Kriegsausgang waren und welche Weichenstellungen sich daraus für die Epigonen ergeben konnten - Athens faktische Niederlage und deren Interpretation durch griechische Intellektuelle bildeten so ein Amalgam, das nur in dieser Verschränkung so vieles anstoßen konnte. Spätere Ausdeutungen und Analogien wurden so präfiguriert, da sie sich vornehmlich aus der vorgeblich eindeutigen Bewertung der Ereignisse durch die griechischen Vorbilder speisten. Etwa finden wir die Auseinandersetzung um die athenische Machtpolitik im Kontext der amerikanischen Unabhängigkeitsbestrebungen wieder, ${ }^{1}$ ebenso um die kluge Ausgestaltung eines British Empire, ${ }^{2}$ Romane entstanden, die das Thema der in den Staub gesunkenen Stadt aufgriffen, ${ }^{3}$ und sogar hochaktuell vermag der ehemalige griechische Finanzminister Varoufakis das Verhalten der Athener als Analogie für das deutsche Vorgehen in der Eurozone zu verwenden, ${ }^{4}$ selbstredend im Blick auf das mögliche Scheitern der von ihm als gefährlich eingestuften Politik.

Doch nicht allein tagespolitisch relevante Akteure, Literaten oder Kommentatoren setzten sich auf diese Weise mit der athenischen Niederlage von 404 auseinander. Derartige Interpretationen wurden durchaus auch von Größen der Wissenschaft vertreten, die auf diesem Wege zu einer gewichtigen Stimme der jeweiligen politischen Argumentation werden konnten. Hier soll daher einer der prominentesten Vertreter der deutschen Altertumswissenschaft im Mittelpunkt stehen, der die Niederlage Athens in besonders exponierter Form zum exemplum für die Situation machte, in der sich aus seiner Sicht Deutschland nach dem Ersten Weltkrieg befand. Die Rede, die Eduard Meyer am 15. Oktober 1919 zum Anlaß seiner Ernennung zum Rektor der Friedrich-WilhelmsUniversität vor Studenten, Kollegen und der interessierten Öffentlichkeit hielt (und die 1924 im zweiten Band der Zweitauflage seiner Kleinen Schriften veröffentlicht wurde), ${ }^{5}$ zeigt die Bildung

(1) Die Frage der Unabhängigkeit der athenischen Bündner und Kolonien wurde als wesentliche Analogie diskutiert, etwa von William Barron, John Simmons and William Meredith, s. dazu z.B. Ceserani 2012, 109; im House of Lords wurde 1777 unter Rückgriff auf die historische Vorlage das eigene Vorgehen gegenüber den amerikanischen Kolonien debattiert, s. The Parliamentary Register Vol. X (1777), 90f.

(2) Dazu bald Benjamin Earley im Band Thalassokratographie - Rezeption und Transformation antiker Seeherrschaft (Hgg. Kopp, H./ Wendt, C.), vorauss. Berlin 2018.

(3) Besonders explizit zu sehen in Callias - A Tale of the Fall of Athens von Alfred John Church (Meadville 1891); s. auch den Beitrag von Dominique Lenfant in dieser Ausgabe, über die Verwendung der antiken Vorlage in der französischen Résistance.

(4) Varoufakis' Buch trägt einen dem Melier-Dialog entlehnten Titel: And the Weak Suffer What They Must, VAROUFAKIS 2016.

(5) Meyer 1924b. 
ganz erstaunlicher Analogien zwischen Berlin/Preußen/Deutschland und dem antiken Athen zum Zwecke der Erhärtung einer politischen und kulturellen Botschaft, die sich aus dem Bild des glorreichen und gestrauchelten Athen speiste und Hoffnung für die Zukunft erwecken sollte.

DER REDNER DES 15. OKTOBER 1919

Zum Zeitpunkt, da Eduard Meyer die soeben angeführte Rede hielt, war er 64 Jahre alt, einer der anerkanntesten Wissenschaftler Europas mit engen Kontakten in die größten Zentren der akademischen Welt. Er hatte Ehrendoktorate der Universitäten Oxford, St. Andrews, Harvard und Chicago inne, die er allerdings wenige Jahre nach ihrer Verleihung aufgrund seiner Verbitterung über die antideutsche Politik der Entente und einige aus seiner Sicht unziemliche Anwürfe an Deutschland niederlegte bzw. gar mit dramatischer Geste zerrissen haben soll. ${ }^{6}$ Anläßlich eines längeren USA-Aufenthalts war ihm zehn Jahre zuvor von Seiten der Presse die Würdigung zuteil geworden, als "the most eminent living historian“ oder "the one final authority“ bezeichnet zu werden, ein Eindruck, der offenbar durch seine gut besuchten öffentlichen Vorträge durchaus nicht in Frage gestellt wurde. ${ }^{7}$

Auch in England, in dem Meyer während des Weltkriegs den deutschen ,Todfeind' ausgemacht hatte, ${ }^{8}$ wurde er intensiv wahrgenommen, ${ }^{9}$ am prominentesten wohl durch Arnold Toynbee, der die Grundidee seines einheitlichen Geschichtsmodells auf die Beschäftigung mit dem Meyerschen Werk zurückführte. ${ }^{10}$ Auch weitere wichtige Theoretiker wie etwa Eric Voegelin wurden auch durch den Kontakt zu Meyer an die Antike herangeführt bzw. durch sein Geschichtsdenken geprägt. $^{11}$

Seine Forschung war ebenfalls global ausgelegt: Der Universalist unter den deutschen Altertumswissenschaftlern bemühte sich früh um einen ganzheitlichen Ansatz des Altertumsverständnisses - die großen altorientalischen Kulturen, Ägypten, das Judentum, all diese wurden wegweisend von ihm in seine Gesamtdarstellung der monumentalen Geschichte des Altertums einbezogen, wie er auch manche bedeutende Detailstudie vorlegte, was ihm insbesondere durch seine stupende Sprachkenntnis ermöglicht wurde. Dies trug ihm überwiegend große Bewunderung, indes auch Kritik von Fachkollegen ein, die manchen großen Bogen Meyers als im Detail nicht aufrechtzuhalten und daher zu oberflächlich ablehnten. ${ }^{12}$

(6) So die Nachricht der Berliner Hochschul-Nachrichten vom 16.2.1920, s. Sösemann 1990, 470.

(7) S. Chambers 1990 zu den amerikanischen Auftritten Meyers und dort zitiert der Evanston Index vom 12. Februar 1910, der zuvor von Meyers Vorträgen als „the literary event of the year“ gesprochen hatte, 97; als Künstler der Formulierung galt Meyer nicht unbedingt, s. den Nachruf von EHrenberg in der HZ 1931, 507: „der einzige der großen deutschen Historiker, dem es nicht gegeben war, seinen Stoff sprachlich wahrhaft zu gestalten“, zudem ist von "nüchternem“, „uncharakteristischem“, an anderer Stelle auch „plumpem“ Deutsch die Rede.

(8) Sösemann 1990, 458f., mit weiteren Nachweisen.

(9) Gilbert Murray unterhielt enge Kontakte nach Berlin (wenn auch primär zu Wilawomitz), s. CALder 1991; Alfred Zimmern studierte 1902 für kurze Zeit an der Berliner Universität, s. etwa MoRefield 2005, 66.

(10) Toynbee 1954, 233.

(11) Voegelin besuchte Meyers Veranstaltungen in Berlin im Jahr 1922/23; seine Erinnerungen sind voll der Bewunderung und unterstreichen explizit den Einfluß, den Meyers Ansatz auf Voegelin ausübte, VoegeLIN 2006, 42f.

(12) S.z.B. den rühmenden Nachruf Ehrenbergs in der HZ, Ehrenberg 1931, 503: „der erste wirkliche Universalhistoriker des Altertums“ u. 502: „ein kyklopisches Mauerwerk, riesig, ungefügt und ungefüge, von grandiosem Willen und souveränem Geist getürmt und gestaltet“; vorsichtig Wilamowitz im Briefwechsel Mommsen/Wilamowitz 2003, Nr. 389, 630f.: ,ich hoffe doch, die Freude an dem Detail, der Einzeluntersuchung, wird ihm noch kommen“, bei insgesamt versöhnlicher Tendenz. 
Doch seine grundlegende Prägung hatte er offensichtlich bereits während seiner Schulzeit an der berühmten Hamburger Gelehrtenschule Johanneum erfahren, wo er unter anderem bei Johannes Classen und Franz Wolfgang Ullrich einen qualitativ höchstwertigen philologischen Unterricht genoß $;{ }^{13}$ neben anderen Interessen wurde hier wohl auch durch diese Spezialisten seine Hochschätzung des Thukydides begründet, ${ }^{14}$ die sein ganzes Leben anhalten sollte. Meyer gilt als einer der sogenannten ,deutschen Thukydideer ${ }^{6}{ }^{15}$ und seine Äußerungen über den Athener lassen keinen Zweifel an der Ausnahmestellung zu, die dessen Werk in Meyers Kosmos einnahm.

Zitiert sei nur die abschließende Passage aus seinem Aufsatz „Zur Theorie und Methodik der Geschichte“ aus dem Jahr 1902, die pars pro toto stehen kann und ähnlich auch in der im folgenden genauer zu untersuchenden Rede wiederholt wird:

„trotzdem gibt es nach wie vor nur eine einzige Art der Geschichte und der Behandlung historischer Probleme, diejenige, welche der Athener Thukydides zuerst geübt und deren Vorbild er in einer von keinem seiner Nachfolger erreichten Vollkommenheit hingestellt hat. ${ }^{\text {"16 }}$

Meyers Umgang mit diesem seinem Vorbild im Kontext des Ersten Weltkriegs soll in den folgenden Überlegungen näher beleuchtet werden, um zu unterstreichen, wie eng die Anlehnung an den athenischen Autor gesucht wurde und wie weitgehend dabei gleichzeitig die Transformation des klassischen Textes geriet.

MIT DER SEELE SUCHEND: EINE REDE ALS BESCHWÖRUNG GEMEINSAMER GRÖSSE

In tiefer Verzweiflung über den Zusammenbruch des Deutschen Kaiserreichs, die Niederlage im Krieg und den Niedergang deutscher Größe übernahm Meyer, der wie Wilamowitz (und Perikles) einen Sohn im Krieg verloren hatte, ${ }^{17}$ die Aufgabe, die Friedrich-Wilhelms-Universität in den schweren Zeiten nach dem Krieg zu leiten. Seine Rektoratsrede vom Oktober 1919 konnte dabei programmatisch verstanden werden, denn er begann sein Amt mit einer inhaltlichen Hinwendung an die Alte Geschichte, die gleichsam zur Aufarbeitung und Analyse des Erlebten dienen wie auch Wege in ein mögliches zukünftiges Leben aufzeigen sollte. Der Universität, wie zu zeigen sein wird, kam in diesem Manifest eine besondere Rolle zu.

Die Parallele zur Niederlage Athens nach dem Peloponnesischen Krieg dient Eduard Meyer in diesem Zusammenhang als die notwendige Analogie, um seiner Erhebung nach erfolgter Feststellung der Ursachen des Niedergangs die Perspektive auf eine bessere Zukunft folgen zu lassen.

Meyer leitet ein, daß, wiewohl der Zusammenbruch Deutschlands „ohne Gleichen in der Weltgeschichte" sei, doch über dem 404 v.Chr. im Peloponnesischen Krieg unterlegenen Athen „ein ähnliches Schicksal geschwebt“ habe, ${ }^{18}$ also eine grundsätzliche Vergleichbarkeit der Konstellationen gegeben sei. Dies gelte im besonderen, so der Althistoriker, da die Feinde in beiden Fällen, also im Umgang mit dem geschlagenen Athen wie dem Deutschen Reich nach der Kapitulation, an der Zerschlagung und der Niederhaltung der bezwungenen Gegner orientiert waren. Diese Grundidee, also die parallele Betrachtung bzw. die Herausstellung der Gleichartigkeit

(13) Zum schulischen Umfeld Meyers Hoffmann 1990, 212-216.

(14) So auch SchLESIER 1990, 380 A.46, und grundsätzlich, wenngleich nicht ohne polemische Töne, zum Verhältnis Meyers zu Thukydides 380-6.

(15) So die Kapitelüberschrift bei MeIster 2013, 12. Kapitel.

(16) Meyer 1924a, 67.

(17) S. Calder 1990, 49; zur Aktivität Meyers im Krieg und seiner Einmischung in die politische Debatte v. UnGERNSternberg 1990 u. Sösemann 1990.

(18) Meyer 1924b, 541 u. 544. 
der historischen Gegebenheiten, verfolgt Meyer daraufhin durch seinen gesamten Redetext, und seine Argumentation beruht entscheidend auf eben dieser angenommenen Analogie, die hier weit mehr ist als gelehrte Ornamentik.

An dieser Stelle soll nur auf einen Teil der Meyerschen Parallelen näher eingegangen werden. Der 29 Seiten starke veröffentlichte Redetext ist mithin ein regelrechtes Sammelbecken an impliziten wie expliziten Bezügen auf das antike Vorbild, die in ihrer direkten Anwendung auf die Gegenwart verblüffen. Anhand dreier Aspekte soll hier nicht dieser Fülle begegnet, sondern die Meyersche Konstruktion in verdichteter Form beleuchtet werden.

\section{a/ Athens und Preußens Gegner}

Ein schlichter und dabei wesentlicher Faktor sind für Meyer die sich im wesentlichen identisch präsentierenden Antinomien, die die Situation nach den Niederlagen Athens 404 und Deutschlands 1918 prägten. Die Unterlegenen über die gleichen Motive ihrer jeweiligen Gegner zu verbinden, als Schicksalsgenossen vorzustellen ist dabei des Redners zentraler Ansatz, der es ihm in weiterer Entwicklung seines Gedankenaufbaus schließlich erlauben soll, ihre Verwandtschaft auch in weiteren Bezügen herzustellen und darüber eine aus der Analogie zu folgernde Botschaft $\mathrm{zu}$ formulieren.

Athen und Preußen/Deutschland sehen sich also dem Althistoriker zufolge einer gleichen feindseligen Gemengelage ausgesetzt. Die Verlogenheit und Berechnung der Siegermächte des Ersten Weltkriegs sind dabei im besonderen das Ziel Meyers: Das Moment des Umgangs mit dem Sieg sowie der propagierten Idee eines Weltfriedens nach dem mörderischen Konflikt zeichnet er als eine genaue Parallele zu der politischen Programmatik der athenischen Gegner, die mit dem Versprechen der Verteidigung von Autonomie die athenische Seite ins Unrecht stellten - „ein Programm", so Meyer,

„das sich niemals verwirklichen konnte, sondern in den Formen eines Gesamtbundes zur Ersetzung der Zwangsherrschaft Athens durch die noch viel umfassendere und weit drückendere Zwangsherrschaft Spartas führen mußte “. ${ }^{19}$

Meyers intendierte Parallelen zum Projekt des Völkerbundes sind hier implizit zu erkennen, ${ }^{20}$ eingangs der Rede jedoch hatte er bereits die Referenz hinsichtlich der Täuschungsabsichten der Feinde etabliert: Das deutsche Volk habe sich

„wie ein Träumender blenden lassen durch die gleißnerischen Verheißungen seiner Todfeinde und durch das Wahngebilde einer greifbar bevorstehenden radikalen Umwandlung des ganzen Menschengeschlechts, einer goldenen Zeit, in der nicht mehr die Macht herrscht, sondern die Gerechtigkeit“. ${ }^{\text {. }}$

Das in Meyers Zeichnung von allen Seiten mit nur vorgespiegeltem und dabei falschem Ethos bedrängte Athen wird also bereits in der grundlegenden Akteurskonstellation zum gegebenen Identifikationspunkt für die Deutschen erhoben, da es ungerechtfertigt und arglistig von der vermeintlich besseren Seite ins Unrecht gestellt wurde; lange habe der Krieg wie eine

(19) Meyer 1924b, 544.

(20) Meyer geht auch an anderer Stelle (und in ähnlicher Diktion) auf die falschen und naiv übernommenen Hoffnungen auf das Wirken einer Völkergemeinschaft ein, z.B. MeYer 1924d, 570 u. 587; zum Engagement einiger Fachkollegen, insbesondere Alfred Zimmern, s. etwa RICH 1995. Ob diese hier angesprochen sind, ist nicht direkt ersichtlich. Daß Zimmern seiner eigenen Kriegspublizistik das berühmte Thukydides-Zitat des Kriegs als ßíaı $\delta\llcorner\delta a ́ \sigma \kappa a \lambda o \varsigma$ $(3,82,2)$ voranstellt (Vorblatt in Zimmern 1919), ist womöglich eine zufällige Parallele zu Meyers Umgang mit dem Athener; zu ähnlichen Ansätzen bei Thibaudet und Toynbee s.u.

(21) Meyer 1924b, 542. 
„Notwendigkeit“ über Athen - und damit auch dem Kaiserreich - geschwebt. ${ }^{22}$ Die Entscheidung, ihm nicht auszuweichen, von Perikles in seiner Kriegsrede des ersten Buches der Geschichte des Peloponnesischen Krieges in extenso dargelegt, ${ }^{23}$ wird in Antike wie Neuzeit zum richtigen, klarsichtigen, staatsmännischen Handeln, das die „Demütigung“ auf der Basis eines klaren Blicks für die Erfolgsaussichten zu umgehen hatte.

\section{b/ Schuld und Schuldige}

Der reale Zusammenbruch der deutschen Ambitionen wird von Eduard Meyer allerdings nicht vornehmlich auf die Stärke oder die Niedertracht der Kriegsgegner (oder gar das Schicksal) zurückgeführt, sondern vielmehr in den begangenen Fehlern und den zu erkennenden Unzulänglichkeiten der deutschen Politik begründet, die er in reichlicher Schonungslosigkeit benennt. Interessanterweise sind dies Faktoren, die sich ihm insgesamt als die gleichen präsentieren, die bereits Athen in dessen Niederlage führten.

Hier ist zunächst die maßlose Hybris zu nennen, oder, wie Meyer es formuliert: „Die Überhebung, zu der wir uns nicht selten haben verleiten lassen, ist uns gründlich ausgetrieben ". ${ }^{24}$ Das Echo vieler Quellen für die athenische Einsicht in ihre eigene Verblendung angesichts ihrer vermeintlichen Machtfülle läßt sich hier erkennen, und Meyer selbst charakterisiert zuvor die athenische Politik wie folgt:

„In dem Gefühl, den feindlichen Ansturm siegreich überstanden zu haben, griff die athenische Demokratie nach Zielen, die sich niemals erreichen ließen. In dem Glauben, jetzt die Herrschaft über ganz Hellas erringen zu können, verfeindete sie sich mit aller Welt, ohne dann auf den einzelnen Schauplätzen mit ausreichender Macht aufzutreten. ${ }^{\text {(25 }}$

Die realpolitische Blindheit also, gepaart mit einem unbegründeten herrschaftslegitimierenden Überlegenheitsgefühl, wird von Meyer als verbindendes Element ausgemacht, das die Niederlage sowohl Athens wie auch Deutschlands zu einem großen Teil bedingte. Vor allem aber konstatiert Eduard Meyer als entscheidenden Grund für den Untergang die für Griechenland und Athen wie Deutschland geltend gemachte innere Zwietracht, die bereits für Thukydides der entscheidende Faktor für Athens Niederlage war. ${ }^{26}$ Diese Bedrohung wird zunächst in grundsätzlicher Manier für die nationale Ebene diagnostiziert:

„Ein gewaltiger Feind freilich steht unüberwunden in unserem Innern, derselbe, dem die griechische Nation erlegen ist, die Zersplitterung und die zentrifugalen Tendenzen. “27

Damit greift Eduard Meyer die Konstellation des Bruderkampfes im Großen auf und verortet in einer - logisch wenig überzeugenden - Transposition Deutschlands Grundproblem in der Antinomie der Partikularinteressen, die allerdings nicht nur, in seiner Diktion, die griechische Nation entscheidend geschwächt hatte, sondern auch Athen in seinem Innern:

„Die Schuld, daß es anders gekommen ist, trägt die Unfähigkeit der athenischen Politik, die Begehrlichkeit der Massen, denen für das Erreichbare das richtige Augenmaß fehlte, die innere Zerrissenheit und das Fehlen eines überlegenen und nicht von Parteileidenschaft und von selbstsüchtigen Bestrebungen beherrschten, sondern von wahrem Patriotismus beseelten, unbeirrt nur den Interessen der Gesamtheit dienenden Staatsmanns. ${ }^{\text {"28 }}$

(22) Ibd., 551.

(23) Thuk. 1, 140-144.

(24) Meyer 1924b, 556.

(25) Ibd., 545.

(26) Thuk. 2,65,11f.

(27) Meyer 1924b, 559.

(28) Meyer 1924b, 551f. 
Und an anderer Stelle liefert Meyer auch die Konkretion seines letzten Gedankens: „Aber einen solchen Staatsmann besaß Athen seit dem Sturz des Perikles nicht mehr, so wenig wie Deutschland nach dem Sturze Bismarcks. ${ }^{\text {29 }}$

Die vorgebliche Vergleichbarkeit der politischen Konstellation also ermöglicht Eduard Meyer die Orientierung an Athen und die Übernahme immerhin einiger Versatzstücke aus dem Analyseschatz des thukydideischen Werks. Dies spitzt er im Hinblick auf das aus den Fugen geratene Verhältnis von Innen- und Außenpolitik noch zu:

„So kam Athen in der entscheidenden Krisis seiner Geschichte nicht aus dem Schwanken heraus; die äußere Politik, die immer den Angelpunkt des Staats bildet und der sich in gesunden Verhältnissen die innere Gestaltung unterzuordnen und anzupassen hat, wurde zum Spielball des inneren Parteikampfs, wie so häufig in aller Geschichte und zu unserem Verderben in all diesen Jahren bei uns. “30

Dies alles scheint dem Meyerschen Verständnis insbesondere der Passage Thuk. 2,65 zu entstammen, in weiter Auslegung auf die deutsche Situation bezogen. Doch ein Moment vermag Meyer zu erkennen, das bei aller Ähnlichkeit zur athenischen Situation den Hohenzollernstaat zusätzlich schwächte und das somit, bei allen positiven Idealen, einer effizienten und erfolgreichen Politik erschwerend im Wege stand. Ein für den Redner massiver Fehler im Staatsbau wird in erneut eindrücklicher Konsequenz betont, der Deutschland charakteristisch eignete und es Meyer zufolge seinen Feinden gegenüber ins Hintertreffen brachte. Es ist dies

„die Entartung unserer Bürokratie, die durch ihren geisttötenden Schematismus und ihren endlosen Instanzenzug die Verantwortung aufhebt, ein selbständiges Durchdenken der Aufgaben und einen daraus erwachsenden freien Willensentschluß erstickt, das unfähige Strebertum großzieht, aber eine überlegene Persönlichkeit nicht aufkommen läßt “. ${ }^{31}$

\section{c/ Hoffnung: per aspera ad astra}

Eben dieses Lamento kann direkt überleiten zum dritten Komplex, nämlich Eduard Meyers Vorstellungen, wie eine Zukunft für eine derart gebrochene Gesellschaft aussehen könne. Exakt dem von ihm gebrandmarkten "geisttötenden Schematismus“ setzt er ein Modell des blühenden Geisteslebens entgegen, das nunmehr die Geschicke Deutschlands wieder im Guten prägen solle - dies ist bereits der die Rede einleitende Gedanke, den Meyer dann historisch unterfüttert wieder aufgreift. Fundiert wird dieser Ansatz auf seiner Interpretation des klassischen Athen, die wiederum entscheidend auf dessen glorreichem Bild aus der Leichenrede des Perikles beruht. Kann nahezu die gesamte Rede unter der Folie des berühmten Vorbilds gelesen werden, ${ }^{32}$ soll der spezifische Focus Meyers auf Geist und Kultur hier schlaglichtartig verdeutlicht werden

Wissenschaft und Bildung sind also für Meyer die einzig mögliche Rettung, da sie eine Idee verkörpern und somit die Eigenart, die so entscheidend ist, auch in die Nachkriegszeit tragen können. Bei Meyer ist „das geistige Leben, in dem seine Eigenart sich entfaltet“, der „Ewigkeitswert“ der Nation, und dies zeitigt somit die „heiligste Aufgabe“, „dieses Gut zu bewahren“. ${ }^{33}$

(29) Ibd., 545

(30) S. parallel Meyer in seinem Brief vom 6.4.1918 an Ehrenberg über „Bethmann und Consorten“: „Diese Leute können nicht begreifen, dass die äussere Politik immer das entscheidende ist und die inneren Verhältnisse so eingerichtet werden müssen, wie es diese erfordert.“, Meyer/Ehrenberg 1990, Brief Nr. 46, 101.

(31) Meyer 1924b, 556; als Parallele hochinteressant sind etwa die Ausführungen Alfred Zimmerns in seinem Aufsatz „German Culture and the British Commonwealth“von 1914 (ursprünglicher Titel: „The War and Democracy") hinsichtlich der schematischen Erziehung in Preußen im Vergleich zum britischen System, Vorstellungen, die schließlich in den Vergleich des Deutschen Reichs mit den Athenern des Melierdialogs münden (ZIMMERN 1919, 19).

(32) So bereits v. Ungern-Sternberg 1990, 501.

(33) Meyer 1924b, 540. 
Dabei paart er eine strenge Hingabe an die Bildung einerseits mit der freien Entfaltung des Einzelnen und seines Geistes als dem Ziel einer Gesellschaft, dies ein Motiv, das wie ein Mantra wiederholt wird. Die Freiheit und die selbsttätige Beteiligung der sich Bildenden wird in direkte Parallele mit den Bürgeridealen gesetzt, die der thukydideische Perikles als die Basis der Stärke seiner Polis aufgezählt hatte. Preußen erscheint in diesem Zusammenhang als das wiedergeborene Athen, da allein hier verstanden worden sei, wie ein Staat auf eben diese Ideale hinzuwirken habe: „Auch auf diesem Gebiet unterscheidet sich unsere Auffassung fundamental von der der Angelsachsen dadurch, daß wir die Pflege der Kultur und des geistigen Lebens als eine der wichtigsten Aufgaben der Gesamtheit und ihres Organs, des Staats, betrachten. “34

Es fiele keinesfalls schwer, für Angelsachsen „andere“ wie bei Thukydides oder direkt „Spartaner“ zu lesen - die Rollenverteilung wird erneut immerhin dem Eingeweihten klar, der den Tenor der perikleischen Leichenrede in den Meyerschen Ausführungen wiedererkennt.

Doch nicht nur in stillschweigend konstruierten Parallelen und codierten Hinweisen, auch ganz explizit stellt Eduard Meyer diese Traditionslinie her, wenn er betont, „den kulturellen Leistungen Athens in seiner Blütezeit“ könne „einzig die Pflege der Wissenschaft zur Seite gestellt werden, die der preußische Staat seit 1808 ununterbrochen geübt hat “ ${ }^{35}$

Und damit schlägt Meyer endlich den Bogen zu seiner eigenen Aufgabe als Rektor der FriedrichWilhelms-Universität, die er als Verwaltung eines großen Erbes ansieht: Der Abqualifikation insbesondere angelsächsischer Universitäten mit ihrem angeblich „mechanischen Abrichten “ und „äußeren Drill ${ }^{\text {“36 }}$ stellt Meyer die Überlegenheit des deutschen Modells samt einer auf die Freiheit, das Engagement und die Verantwortung der Studenten für das Ganze ausgerichteten Konzeption entgegen. Daher seien es die deutschen Universitäten, die zum geistigen Wiederaufbau berufen seien, da sie als einzige in ihrem ,idealen Gehalt unerschüttert" geblieben seien. ${ }^{37}$ Wenn sie also schon nicht die Schule von Hellas sein können, so verkörpern die höheren Bildungsanstalten in Meyer'scher Deutung in nuce alle idealisierten Werte, die Thukydides Perikles so suggestiv betonen ließ. ${ }^{38}$

Preußen - oder besser: das in der Idee der Universitäten lebende Preußen $-^{39}$ wird somit in Meyers Konstruktion zum wahren Träger des athenischen bzw. perikleischen Ideals, und die schreckliche Situation nach dem Weltkrieg ist letztlich, bei allem Bedauern über den Ernst der Lage, primär Katalysator für die Besinnung auf die große Aufgabe, die bereits Athen so überzeugend gemeistert hatte. Die Weltgeltung, das Unvergängliche Athens lebt in der preußisch-deutschen Kulturauffassung, und berufen zur Nachfolge der Stadt, deren Name „vom Glanze umstrahlt ist wie der keiner anderen Stadt auf Erden", ${ }^{40}$ scheint, in einer spezifischen translatio-Vorstellung Meyers, Spree-Athen, Berlin zu sein. Die offen eingestandene exklusive Beanspruchung des athenischen Vorbilds bzw. der Seelenverwandtschaft Athens und Berlins ist dabei der Kunstgriff, der analog zur offenbar von Meyer angenommenen Intention der Perikles-Rede, nämlich als nachträgliches Elogium auf das untergegangene Athen, funktioniert: „Diese gewaltigen Leistungen Athens haben

(34) Ibd., 561.

(35) $\mathrm{Ebd}$.

(36) Beide Zitate Meyer 1924b, 564.

(37) Ibd., 541.

(38) Dieser Aspekt findet sich auch wieder in Meyers Ideal der „freien Unterordnung“ unter Satzungen und Schranken (1924b, 564), das wie Thuk. 2,37, also dem Bild des freien Bürgers, der die Gesetze in Freiheit achtet und fürchtet, entnommen scheint.

(39) Hierin ist Momiglianos Deutung zu schematisch, der Meyers Haltung grundlegend als unverbrüchliche Treue zum alten Preußen deutet, Momigliano 1984, 230.

(40) Meyer 1924b, 555; s. parallel 552: „ihm bleibt der unvergängliche Ruhm, durch den es alle andern Staaten der Welt überragt". 
es dem Schriftsteller ermöglicht, eben da es gefallen war, ein Idealbild seines inneren Wesens zu zeichnen." ${ }^{\text {41 }}$

Meyer entwirft dabei die vergangene Größe Preußens/Deutschlands bis in die Diktion an den thukydideischen Perikles angelehnt, und in dieser Inanspruchnahme scheint es, als sei die Leichenrede des Perikles im Grunde vorausschauend für die Friedrich-Wilhelms-Universität zu Berlin verfaßt worden. ${ }^{42}$ Wie wesentlich die eigene Orientierung am thukydideischen Muster für Eduard Meyer war, belegt die Tatsache, daß ihm das Vorbild sowohl in wissenschaftlicher und geschichtstheoretischer Hinsicht als entscheidend vor Augen stand, wie auch in dessen politischer Inanspruchnahme, nämlich zwecks der Schaffung einer möglichst unanfechtbaren Autorität für seine, Meyers Aussagen. Thukydides fungiert hier wie ein sicheres Fundament, auf dem eine politische Argumentation überzeugend beruhen kann.

Ganz explizit verfährt Meyer schließlich mit seinem großen Vorbild, indem er die Aktualität des Thukydides beschwört:

„Wenn zertrümmert am Boden liegt und mit Füßen getreten wird, was uns heilig war, so wollen wir dennoch mit Thukydides bekennen: es war doch etwas Gewaltiges, was unser Volk geschaffen und erstrebt hat und wofür es gelitten hat und noch leidet." ${ }^{\text {"43 }}$

Dies ist die Klimax des gesamten Textes, denn hier ist die Parallele auf die Spitze getrieben, die Grenzen zwischen Antike und Neuzeit verwischen endgültig, und Thukydides (als vermeintlicher Apologet der Größe Athens) wird offiziell zum Kronzeugen der Größe der deutschen Errungenschaften. Angesichts der Möglichkeiten, die der thukydideische Text anbietet, ihn als Vorgriff auf die politische Menschheitsgeschichte zu lesen, ist dieses zunächst abstrus anmutende intellektuelle Vorgehen letztlich nichts als die konsequente Jüngerschaft seitens derer, die in Thukydides ihren allwissenden Meister erkannt zu haben meinten. Dabei ist diese sehr spezielle Form des ,Thukydidismus', ${ }^{44}$ also hier eines sehr pragmatisch verstandenen Anspruchs auf Anleitung ${ }^{45}$ und überzeitliche axiomatische Geltung des alten Textes, nicht einmal sonderlich originell oder neu, ${ }^{46}$ aber sie nimmt im 20. Jahrhundert, zumal nach dem Erlebnis des Ersten Weltkriegs, rasant zu. Albert Thibaudets La campagne avec Thucydide oder Arnold Toynbees Erinnerungen an seine Thukydides-Lektüre im Jahr 1914 sind zwei besonders sprechende und im Ansatz an Eduard Meyer erinnernde Beispiele. Wo Thibaudet in seinem siebten Kapitel „Les deux guerres" die Vergleichbarkeiten der Konflikte postuliert und hinterfragt, um aus Thukydides' Werk eine bei aller Differenz der Zeiten eminent aktuelle und für die Neuzeit aussagekräftige Analyse zu machen, ${ }^{47}$ geht die Wahrnehmung bei Toynbee gar noch weiter, wenn er die Zeitgenossenschaft des Thukydides offen beansprucht:

(41) Ibd., 553

(42) Damit ist Meyers Rede kein dem Epitaphios rein formal analoger Nachruf (so aber v. Ungern-STERnBERg 1990, 502), sondern vielmehr ein aus dem Grundsatz des Thukydides, einen überzeitlichen Besitz anzubieten, schöpfender Versuch, das sehr Lebendige des preußisch-perikleischen Wertekanons zu betonen.

(43) Meyer 1924b, 556.

(44) $\mathrm{Zu}$ diesem Begriff s. Meineke 2003.

(45) SChlesier 1990, 384f.; allg. zu solchem Verständnis des Thukydides bis heute s. OвER 2006; Wendt 2016.

(46) $\mathrm{Zu}$ Thukydides bei Machiavelli, Hobbes, Adams, Lévesque und vielen weiteren Inanspruchnahmen existiert eine Fülle an Spezialliteratur, s. als Überblick nur LeE/MorLey 2015.

(47) Thibaudet 1922 zeichnet die Identifikationen interessanterweise diametral von Meyer abweichend: die Seemacht Athen sieht er im Vereinigten Königreich vertreten, während Berlin wie Sparta (234) auf die Überlegenheit seiner kontinentalen Ressourcen vertraut. Die Ähnlichkeiten der athenischen Kapitulation mit dem Waffenstillstandsabkommen des 11. November 1918 aber ermöglicht auch ihm die Gleichsetzung der athenischen Ambitionen („le rêve démesuré d'un empire athénien“) mit der Situation des Deutschen Reichs (238). Auch auf systemischer Ebene zieht Thibaudet enge Parallelen: „La grande guerre laissera un monde européen pas très différent du monde grec que laissait la guerre du Péloponnèse." (253) 
„The experience that we were having in our world now had been experienced by Thucydides in his world already. I was re-reading him now with a new perception - perceiving meanings in his words, and feelings behind his phrases, to which I had been insensible until I, in my turn, had run into that historical crisis that had inspired him to write his work. Thucydides, it now appeared, had been over this ground before. He and his generation had been ahead of me and mine in the stage of historical experience that we had respectively reached; in fact, his present had been my future. But this made nonsense of the chronological notation which registered my world as ,modern' and Thucydides' world as ,ancient'. Whatever chronology might say, Thucydides' world and my world had now proved to be philosophically contemporary. “48

Eduard Meyer stand also mit seiner grundsätzlichen Anwendung des vermeintlichen thukydideischen Paradigmas in seiner Zeit keineswegs allein, ${ }^{49}$ und es ist letztlich die Auslegung und die spezifische Anverwandlung des Atheners, in der er eine eigene und höchst eigenwillige Position einnahm. Dies geht so weit, daß an mancher Stelle Thukydides und Meyer eine eigentümliche Symbiose eingehen, Meyers Thukydides deutliche Züge Meyers selbst trägt, als sei über die Erkenntnis der historisch treffenden Analogie auch die Seelenverwandtschaft mit dem Athener noch inniger geworden. ${ }^{50}$

Doch ist es hilfreich, daran zu erinnern, daß Eduard Meyer die Situation der Weltpolitik keineswegs immer mit griechischen oder gar athenischen Analogien zu verdeutlichen suchte - mindestens ebenso prominent, wohl sogar häufiger bemüht, ist die Folie des römischkarthagischen Konflikts (sogar kurz in der hier behandelten Rede) ${ }^{51}$, wobei dieser insgesamt wesentlich illustrativer gebraucht wird als die in der Rektoratsrede evozierte tiefe Wesensgleichheit von Deutschen/Preußen und Athenern; Meyer tauscht auch durchaus die Zuschreibungen aus, wenn ihm an der griffigen Plastizität seiner Beispiele gelegen ist, Hannibal etwa mal auf englischer, mal auf deutscher Seite zum Einsatz kommt. ${ }^{52}$ Und auch eine Antikenfixiertheit kann Meyer nicht grundsätzlich attestiert werden, bedenkt man, wie häufig er sich ohne den Umweg über die Alte Welt direkt gegen politische Gegner und als solche wahrgenommene ,Todfeinde' geäußert hat. ${ }^{53}$ Dennoch ist bemerkenswert, in welcher Rolle die antike Tradition ihm jederzeit zu Gebote stehen kann, je nachdem, welche Argumentation mit welcher Autorität versehen an die Adressaten gebracht werden soll.

\section{ANALOGIE UND GESCHICHTSVERSTÄNDNIS}

Die in dieser Verwendung der Antike zum Ausdruck kommende Methode Meyers speist sich aus seiner Auffassung, daß allein das Gegenwärtige das Interesse an der fernen Vergangenheit generiere, wie er, wiederum in „Zur Theorie und Methodik der Geschichte“, ausgeführt hat:

„Auch hier kann die Antwort nur die Gegenwart geben: die Auswahl beruht auf dem historischen Interesse, welches die Gegenwart an irgend einer Wirkung, einem Ergebnis der Entwicklung hat, so dass sie das Bedürfnis empfindet, den Anlässen nachzuspüren, welche es herbeigeführt haben.

(48) Toynbee 1948, 7f.; sehr ähnlich und explizit auf das Erlebnis des Ersten Weltkriegs bezogen ToynbeE 1954, 233.

(49) S. auch v. Ungern-Sternberg 2006, 249-252, zu ähnlichen Tendenzen in der deutschen Forschung, etwa bei Erich Bethe; ebenso Nippel 2016, 308f.; s. auch den Beitrag von Schelske in dieser Ausgabe.

(50) Diese Tendenz kulminiert in Meyer 1924b, 551, wo die Perspektiven zu verwischen scheinen: „das Herz schlägt ihm höher, wenn er an ihre Herrlichkeit und die nun versunkene Machtstellung ihres Reiches denkt. Eben der Untergang dieses Reichs macht es möglich, es von höherem Standpunkt aus, erhaben über die Gegensätze und Parteikämpfe des Tages, in seiner historischen Größe zu würdigen."

(51) Ibd., 543.

(52) Siehe dazu auch v. Ungern-Sternberg 1990, 497-502, mit weiteren Nachweisen.

(53) So auch Nippel 2016, 309. 
Welchen Gebieten sich dieses Interesse in erhöhtem Masse zuwendet, ist von der Gestaltung der Gegenwart abhängig. “54

Die Unterordnung also, die das Material antiker Überlieferung unter das Erkenntnisinteresse des aus der Gegenwart Betrachtenden damit erfährt, ist ein Merkmal, das den Zugriff auf die zu rekonstruierende Vergangenheit über das Mittel der Analogie geradezu einfordert und damit die Antike stets dem Primat der nützlichen Erkenntnis unterwirft, ganz in Abkehr von Ansätzen, die den Idealgehalt der Alten Welt als Leitstern für die Gegenwart zu transportieren suchten.

Werner Jaeger faßte dieses Geschichtsverständnis in einer Gedenkrede auf Eduard Meyer wie folgt zusammen:

„Eduard Meyer war zwar kein Humanist, der der eigenen Zeit die idealen Geisteswerte der Vergangenheit in Kunst und Philosophie als höchste Maßstäbe vorhält. Er war ganz historischer Realist, und die Quellen lesen, das hieß für ihn: rücksichtlos die geistige Form, in der die Geschichte überliefert ist, wie eine Maske abstreifen und sie in die Realität des noch gärenden Lebensvorgangs zurückübersetzen. " ${ }^{\text {55 }}$

Victor Ehrenberg, selbst ein Schüler Meyers, problematisiert diesen grundsätzlichen Ansatz hingegen in seinem Nachruf in der Historischen Zeitschrift aus dem Jahr 1931:

„In einer Hinsicht allerdings verfiel diese kritische Geschichtsschreibung einem Fehler (hierin Theodor Mommsen, dem sonst bei aller Verehrung mit starken Vorbehalten betrachteten Führer auf römischem Gebiete, verwandt), einem Fehler, der gerade einer kritischen Grundeinstellung widersprach: daß man nämlich die eigene Zeit allzu sehr in die Antike hineinsah. ${ }^{\text {“56 }}$

Dies aber ist keineswegs ein Versehen oder eine methodische Nachlässigkeit Meyers, sondern es steht exemplarisch für eine neue Lesart bzw. eine neue Verinnerlichung des thukydideischen Vorsatzes, etwas Nützliches für alle, die klar sehen wollen, und damit einen Besitz für alle Zeit für die Erkenntnis der im Kern konstanten menschlichen Natur zu schaffen; Thukydides also als Maßstab oder gar Anleitung für die Bewertung der Gegenwart zu verstehen ist dementsprechend kein ausschließliches Produkt der Politikwissenschaft (aus der wir solches bis heute kennen) ${ }^{57}$, sondern eine nicht zuletzt genuin altertumswissenschaftliche Annäherung an seinen Gehalt. Die Anläufe der Schule von Leo Strauss etwa, die in der Geschichte des Peloponnesischen Krieges einen dekontextualisierungsfähigen, überzeitlich gültigen Text sehen, der gerade nicht als der eines modern verstandenen Historikers gelesen werden sollte, ${ }^{58}$ sind trotz einer disziplinär etablierten Distanz im Kern von einer Verwendung wie der durch Eduard Meyer nicht weit entfernt. Dabei kommt es schließlich weniger darauf an, mit welchem Genreverständnis man sich dem thukydideischen Werk nähert, als vielmehr, wie hoch man die im Werk formulierte

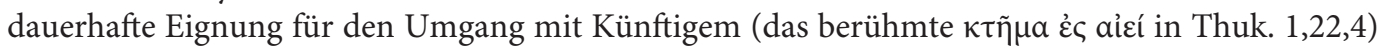
veranschlagt (und welche Hoffnungen auf die anleitende Funktion eines antiken Werks man grundsätzlich hegt) $)^{59}$ - Strauss und Meyer legen eben diese „Anwendbarkeit“ des Thukydides ihren Interpretationen zugrunde.

Wie um einen derartigen Ansatz zu untermauern und zu etablieren, urteilt Meyer grundlegend (und dabei ganz als Epigone des Thukydides) über die Alte Geschichte:

(54) Meyer 1924a, 44.

(55) Zitiert in: CALder 1990, 50.

(56) Ehrenberg 1931, 504.

(57) Hier sei nur auf das aktuellste Beispiel verwiesen, das auf einer langen Tradition fußt: Graham Allisons, Thucydides Trap' (Allison 2017); eine theoretische Grundierung etwa bei Waltz 1959, 210f. u. Gilpin 1988; kritisch dazu z.B. LEBOw 2008.

(58) Strauss 1964, insb. 139-144; aus der Fülle der Literatur zu diesem Aspekt s. jüngst etwa Orwin 2015; JAFFe 2015; Keedus 2016.

(59) Zu diesem Aspekt Morley 2015. 
„wohl aber bietet sie zu ihnen [den Weltkriegen] die mannigfachsten Analogien, wie es denn überhaupt ihre Eigenart ist und ihr ein spezifisches Interesse verleiht, daß dieselben Vorgänge, die uns später in den größeren Dimensionen der neueren Geschichte entgegentreten, hier in den Verhältnissen einer weit enger begrenzten Welt sich abspielen. Eben dadurch, daß sie leichter übersehbar sind und daß sie in ihren Wirkungen völlig abgeschlossen vorliegen, erhalten sie zugleich einen typischen Charakter und geben uns zum Verständnis und zur Beurteilung der späteren Entwicklung einen garnicht hoch genug einzuschätzenden Leitfaden in die Hand. “60

Vor allem sind derartige Aussagen bemerkenswert, wenn man bedenkt, daß Meyer selbst prominent vor den Gefahren der Analogiebildung warnte und ebenfalls die Suche nach ewigen Gesetzen der Geschichte entschieden ablehnte, die der Andersartigkeit, dem Zufall und der Unwiederholbarkeit nicht genug Raum in der Geschichtsbetrachtung gestatteten. ${ }^{61}$ Andererseits bedeutet eine derartige Reserviertheit wohl nicht mehr, als nach demjenigen zu verlangen, der auch die Faktoren der Kontingenz und der individuellen Abweichung mit historisch sicherem Blick erkennen und gewichten kann, um die Analogiebildung qua der treffenden prinzipiellen Abstraktion auf intellektuell akzeptabler Basis zu betreiben.

Denn wie auch Theodor Mommsens Schöpfung der Römischen Geschichte nicht zuletzt der politischen Wahrnehmung ihres Autors entsprang, die Einheit der Nation sei das vordringliche Element der Politik seiner Zeit und damit durchaus das gegebene Paradigma zur Untersuchung und Bewertung der Leistungen Roms, ${ }^{62}$ so ist auch für Meyer der Impuls für seine Rückschau der, Paradigmatisches zu finden, das als Erklärungsmuster oder gar als Orientierungshilfe für die eigene Situation dienstbar gemacht werden kann. Und daß Meyer auf der Suche nach einer solchen Orientierung, sogar nach einer neuen Hoffnung war, kann, wie auch aus Briefen Meyers, ${ }^{63}$ aus dem Vorwort zur Zweitauflage seines sehr erfolgreichen Werks Caesars Monarchie und das Principat des Pompejus geschlossen werden, das wenige Monate vor der Rektoratsrede, im Juli 1919, fertiggestellt wurde:

„Jetzt ist das alles niedergetreten und vernichtet, zahlreiche der edelsten Männer und Frauen sind einem gräßlichen Schicksal anheimgefallen, ein blühendes Kulturland ist der rohesten Barbarei ausgeliefert; und dahinter steht die furchtbare Tatsache, daß dies Schicksal nicht nur durch das Verbrechen unserer Feinde herbeigeführt ist, sondern ein großer Teil der Schuld auf uns selbst lastet, daß unser Volk der großen weltgeschichtlichen Aufgabe, die ihm gestellt war, nicht gewachsen gewesen ist, und daß das gleiche Schicksal jetzt drohend auch über der eigenen Heimat schwebt. Wo ist noch ein Hoffnungsanker, an den wir uns klammern können, um an der Zukunft unserer Nation nicht zu verzweifeln?"64

Es wirkt, ohne sich damit in allzu plumpe psychologische Annäherungen zu verstricken, als habe Eduard Meyer eben den von ihm beschworenen „Hoffnungsanker“ wenige Monate später für seine Zuhörerschaft im klassischen Athen ausgemacht, und als habe er diese Funktion Athens nicht aus den Grundlagen der Griechenlandbegeisterung des gelehrten Humanismus kreiert, sondern als habe es ihm die Situation aufgetragen, ein dringend benötigtes Hoffnung generierendes Beispiel in den ihm vertrauten Überlieferungen zu verorten. Daß dies über die Inanspruchnahme der in seinem über alles geschätzten Thukydides vorzufindenden Rhetorik gelang, ist keine Überraschung; daß hingegen das thukydideische Angebot, seinen Text wie eine

(60) Diese Anschauung findet sich in der Rede „Vorläufer des Weltkriegs im Altertum“, gehalten 24. Januar 1918, in: MEYer 1924c, 512.

(61) S. etwa Meyer 1924a, 32ff.; s. auch v. Ungern-Sternberg 1990, 504.

(62) S. dazu etwa Flaig 2005, bes. 181f.

(63) S. Brief Nr. 48 in Meyer/Ehrenberg 1990, der neben tiefer Hoffnungslosigkeit doch die geistige Tätigkeit und die Wissenschaft als mögliche Rettung benennt, die sich für Meyer jedoch noch nicht eingestellt habe, 104f.

(64) Abgedruckt in Meyer 1922, XIV. 
Folie für kommende Ereignisse und Konstellationen zu gebrauchen, ${ }^{65}$ von Meyer in einer derart zugespitzten interpretatio angenommen wurde, zeugt von Meyers Jüngerschaft ebenso wie von seiner inhaltlichen Vorprägung, die die Quelleninterpretation bekanntlich selten positiv beeinflußt.

Die Niederlage Athens im Peloponnesischen Krieg also war für Eduard Meyer das gegebene Exempel, um einerseits die für ihn aktuelle Konstellation der Weltpolitik zu analysieren und aus den begangenen Fehlern zu lernen, andererseits aber auch, um aus dem Wiederaufstieg Athens im 4. vorchristlichen Jahrhundert die notwendige geistige Aufrüstung zu gewinnen, die eine Neudefinition des Preußisch-Deutschen im Sinne eines wahren Humanismus nach sich ziehen sollte. Keinesfalls lag es Meyer daran, diese Aufgabe als globale Herausforderung und als Besinnung auf ein notwendig gewordenes größeres koเvóv aufzufassen, so wie es andere berühmte Gelehrte seiner Zeit taten. ${ }^{66}$ Im Gegenteil: Das Trotzig-Exklusive, mit dem er Deutschlands Sonderstellung in der Welt nun auf andere, weiterhin aggressive Weise einforderte - hierin der Strategie athenischer Redner im 4. Jh. nicht ganz fremd $-{ }^{67}$ zeugt davon, wie er Thukydides zu einer politischen Tendenzschrift umdeutet, um die Erwähltheit seines Landes und Volkes mittels der antiken Autorität zu legitimieren, ähnlich wie dies der thukydideische Perikles seinen Zuhörern im Hinblick auf Athen suggeriert hatte. Die Thukydides' Text innewohnende Spannung, die heikle Doppelbödigkeit seiner Konstruktionen hatten in einer derartigen Lesart naturgemäß keinen Platz. Wie in Thukydides' Athen indes, und diese Dimension sah Meyer offenbar erst recht nicht, ließ denn auch der Seucheneinbruch in Deutschland nicht mehr lange auf sich warten. ${ }^{68}$

Christian WendT

Freie Universität Berlin

\section{Bibliographie}

Allison, G., 2017, Destined for War. Can America and China escape Thucydides's Trap?, Boston.

Calder, W.M., „Credo gegen Credo; Arbeit gegen Arbeit; Anschauung gegen Anschauung'. Ulrich von Wilamowitz-Moellendorff contra Eduard Meyer", in Eduard Meyer. Leben und Leistung eines Universalhistorikers, ed. Calder, W.M./Demandt, A., Leiden/New York/Kopenhagen/Köln 1990, 41-73.

-, 1991, The Prussian and the Poet. The Letters of Ulrich von Wilamowitz-Moellendorff to Gilbert Murray (1894-1930), Hildesheim.

Ceserani, G., 2012, Italy's Lost Greece: Magna Graecia and the Making of Modern Archaeology, Oxford.

Chambers, M., 1990, „The ,Most Eminent Living Historian, the One Final Authority': Meyer in America“, in Eduard Meyer. Leben und Leistung eines Universalhistorikers, ed. Calder, W.M./Demandt, A., Leiden/ New York/Kopenhagen/Köln, 97-131.

Ehrenberg, V., « Eduard Meyer », Historische Zeitschrift 143 (1931), 501-511.

(65) Zumindest ist dies eine Möglichkeit, Thuk. 1,22,4 zu lesen.

(66) Nochmals sei exemplarisch auf Alfred Zimmerns Publizistik hingewiesen, etwa 1919, 20: „We are still far off from the World-State and the World-Law which formed the misty ideal of cosmopolitan thinkers. But only those who are blind to the true course of human progress can fail to see that the day of the Nation-State is even now drawing to a close."

(67) Als nur ein Beispiel s. Isokrates' Panegyrikos, z.B. 20-9 u. 46-50 u. passim.

(68) Zum berühmten Diptychon Leichenrede - Seucheneinfall Flashar 1969, insb. 34ff. 
Flaig, E., 2005, „Die verfehlte Nation. Warum Mommsens Rom nicht ans geschichtliche Ziel gelangte“, in Theodor Mommsen. Wissenschaft und Politik im 19. Jahrhundert, ed. Demandt, A./Goltz, A./SchlangeSchöningen, H., Berlin/New York, 181-200.

Flashar, H., 1969, Der Epitaphios des Perikles. Seine Funktion im Geschichtswerk des Thukydides, Heidelberg. Gilpin, R., 1988, „The Theory of Hegemonic War“, in The Journal of Interdisciplinary History 18,4, 591-613.

Hoffmann, C., 1990, „Die Selbsterziehung des Historikers: Zur intellektuellen Entwicklung des jungen Eduard Meyer (1855-1879)“, in Eduard Meyer. Leben und Leistung eines Universalhistorikers, ed. Calder, W.M./ Demandt, A., Leiden/New York/Kopenhagen/Köln, 208-254.

Jaffe, S., 2015, „The Straussian Thucydides“, in A Handbook to the Reception of Thucydides, ed. Lee, C./Morley, N., Malden, 278-295.

Keedus, L., 2016, „Leo Strauss's Thucydides and the Meaning of Politics“, in Thucydides and Political Order. Lessons of Governance and the History of the Peloponnesian War, ed. Thauer, C./Wendt, C., New York, 75-93.

Lebow, R.N., 2008, „The Ancient Greeks and Modern Realism: Ethics, Persuasion, and Power“, in: Political Thought and International Relations. Variations on a Realist Theme, ed. Bell, D., Oxford, 26-40.

Lee, C./Morley, N. (ed.), 2015, A Handbook to the Reception of Thucydides, Malden.

Meineke, S., 2003, „Thukydidismus“, in Der Neue Pauly 15/3, ed. Canzik, H./Schneider, H./Landfester, M., Stuttgart, 480-494.

Meister, K., 2013, Thukydides als Vorbild der Historiker. Von der Antike bis zur Gegenwart, Paderborn.

Meyer, E., 1922, Caesars Monarchie und das Principat des Pompejus, Berlin.

—, 1924a, „Zur Theorie und Methodik der Geschichte“, in ders., Kleine Schriften, Bd. 1, 2.A., Halle (Saale), 1-67.

—, 1924b, „Rede beim Antritt des Rektorats der Friedrich-Wilhelms-Universität Berlin am 15. Oktober 1919“, in ders., Kleine Schriften, Bd. 2, Halle (Saale) , 539-567.

—, 1924c, „Vorläufer des Weltkriegs im Altertum. Wissenschaftliche Festrede, gehalten in der öffentlichen Sitzung der preußischen Akademie der Wissenschaften am 24. Januar 1918 zur Feier des Geburtstages Sr. Majestät des Kaisers und Königs und des Jahrestages König Friedrichs II“, in ders., Kleine Schriften, Bd. 2, Halle (Saale), 507-538.

—, 1924d, „Rede zur Gedächtnisfeier des Stifters der Berliner Universität König Friedrich Wilhelms III. am 3. Auguts 1920“, in ders., Kleine Schriften, Bd. 2, Halle (Saale), 568-591.

Meyer, E. und Ehrenberg, V., 1990, Ein Briefwechsel 1914-1930, ed. Audring, G./Hoffmann, C./v. UngernSternberg, J., Stuttgart.

Momigliano, A., 1984, „Premesse per una discussione su Eduard Meyer“, in ders., Settimo contributo alla storia degli studi classici e del mondo antico, Rom, 215-231.

Mommsen, T. und Wilamowitz, U., 2003, „Aus dem Freund ein Sohn“. Theodor Mommsen und Ulrich von Wilamowitz-Moellendorff. Ein Briefwechsel 1872-1903, ed. Calder, W./Kirstein, R., Hildesheim.

Morefield, J., 2005, Covenants without Swords. Idealist Liberalism and the Spirit of Empire, Princeton.

Morley, N., 2015, „Contextualism and Universalism in Thucydidean Thought“, in Thucydides and Political Order. Concepts of Order and the History of the Peloponnesian War, ed. Thauer, C./Wendt, C., New York, 23-40.

Nippel, W., 2016, Ancient and Modern Democracy. Two concepts of Liberty?, transl. Tribe, K., Cambridge.

Ober, J., 2006, „Thucydides and the Invention of Political Science“, in Brill's Companion to Thucydides, ed. Rengakos, A./Tsakmakis, A., Leiden/Boston/Tokyo, 131-59.

Orwin, C., 2015, „Reading Thucydides with Leo Strauss“, in: Brill's Companion to Leo Strauss' Writings on Classical Political Thought, ed. Burns, T., Leiden/Boston/Tokyo, 50-75. 
Rich, P., 1995, „Alfred Zimmern's Cautious Idealism: The League of Nations, International Education and the Commonwealth“, in Thinkers of the Twenty Years' Crisis. Interwar Idealism Reassessed, ed. Long, D./ Wilson, P., Oxford, 79-100.

Schlesier, R., 1990, „Religion als Gegenbild. Zu einigen geschichtstheoretischen Aspekten von Eduard Meyers Universalhistorie“, in Eduard Meyer. Leben und Leistung eines Universalhistorikers, ed. Calder, W.M./ Demandt, A., Leiden/New York/Kopenhagen/Köln, 368-416.

Sösemann, B., 1990, „,Der kühnste Entschluß führt am sichersten zum Ziel. 'Eduard Meyer und die Politik“, in Eduard Meyer. Leben und Leistung eines Universalhistorikers, ed. Calder, W.M./Demandt, A., Leiden/ New York/Kopenhagen/Köln, 446-483.

Strauss, L., 1964, City and Man, Chicago.

Thibaudet, A., 1922, La campagne avec Thucydide, Paris.

Toynbee, A., 1948, Civilization on trial, New York.

-, 1954, A Study in History, Vol. X, Oxford.

v. Ungern Sternberg, J., 1990, „Politik und Geschichte. Der Althistoriker Eduard Meyer im Ersten Weltkrieg“, in Eduard Meyer. Leben und Leistung eines Universalhistorikers, ed. Calder, W.M./Demandt, A., Leiden/ New York/Kopenhagen/Köln, 484-504.

—, 2006, „Deutsche Altertumswissenschaftler im Ersten Weltkrieg“, in Kollegen, Kommilitonen, Kämpfer: europäische Universitäten im Ersten Weltkrieg, ed. Maurer, T., Stuttgart, 239-254.

Varoufakis, I., 2016, And the Weak Suffer What They Must?: Europe, Austerity and the Threat to Global Stability, London.

Voegelin, E., 2006, Autobiographical Reflections. The Collected Works of Eric Voegelin, Vol. 34, ed. Sandoz, E., Columbia/London.

Waltz, K., 2001, Man, the State and War, New York (Erstausgabe 1959).

Wendt, C., 2016, „Thucydides as a ,Statesmen's Manual'?“, in Thucydides and Political Order. Lessons of Governance and the History of the Peloponnesian War, ed. Thauer, C./Wendt, C., New York, 151-167.

Zimmern, A., 1919, „German Culture and the British Commonwealth“, in ders., Nationality and Government, with other war-time essays, new ed. London, 1-31. 